\title{
Optical interferometry and Gaia measurement uncertainties reveal the physics of asymptotic giant branch stars
}

\author{
A. Chiavassa ${ }^{1}$, K. Kravchenko ${ }^{2}$, F. Millour ${ }^{1}$, G. Schaefer ${ }^{3}$, M. Schultheis ${ }^{1}$, B. Freytag ${ }^{4}$, O. Creevey ${ }^{1}$, V. Hocdé ${ }^{1}$,

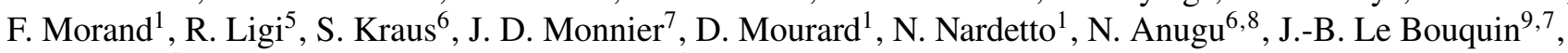 \\ C. L. Davies ${ }^{6}$, J. Ennis ${ }^{7}$, T. Gardner ${ }^{7}$, A. Labdon ${ }^{6}$, C. Lanthermann ${ }^{10}$, B. R. Setterholm ${ }^{7}$, and T. ten Brummelaar ${ }^{3}$ \\ ${ }^{1}$ Université Côte d'Azur, Observatoire de la Côte d'Azur, CNRS, Lagrange, CS 34229, Nice, France \\ e-mail: andrea.chiavassa@oca.eu \\ ${ }^{2}$ European Southern Observatory, Alonso de Cordova 3107, Santiago, Chile \\ 3 The CHARA Array, Mount Wilson Observatory, Mount Wilson, CA 91023, USA \\ ${ }^{4}$ Department of Physics and Astronomy at Uppsala University, Regementsvägen 1, Box 516, 75120 Uppsala, Sweden \\ ${ }^{5}$ INAF - Osservatorio Astronomico di Brera, Via E. Bianchi 46, 23807 Merate, Italy \\ ${ }^{6}$ University of Exeter, School of Physics and Astronomy, Stocker Road, Exeter, EX4 4QL, UK \\ ${ }^{7}$ Department of Astronomy, University of Michigan, Ann Arbor, MI 48109, USA \\ ${ }^{8}$ Steward Observatory, 933 N. Cherry Avenue, University of Arizona, Tucson, AZ 85721, USA \\ ${ }^{9}$ Institut de Planétologie et d'Astrophysique de Grenoble, CNRS, Univ. Grenoble Alpes, Grenoble, France \\ ${ }^{10}$ Instituut voor Sterrenkunde, KU Leuven, Celestijnenlaan 200D, 3001 Leuven, Belgium
}

Received 26 February 2020 / Accepted 3 June 2020

\section{ABSTRACT}

Context. Asymptotic giant branch (AGB) stars are cool luminous evolved stars that are well observable across the Galaxy and populating Gaia data. They have complex stellar surface dynamics, which amplifies the uncertainties on stellar parameters and distances. Aims. On the AGB star CL Lac, it has been shown that the convection-related variability accounts for a substantial part of the Gaia DR2 parallax error. We observed this star with the MIRC-X beam combiner installed at the CHARA interferometer to detect the presence of stellar surface inhomogeneities.

Methods. We performed the reconstruction of aperture synthesis images from the interferometric observations at different wavelengths. Then, we used 3D radiative hydrodynamics (RHD) simulations of stellar convection with CO5BOLD and the post-processing radiative transfer code OPTIM3D to compute intensity maps in the spectral channels of MIRC-X observations. Then, we determined the stellar radius using the average 3D intensity profile and, finally, compared the 3D synthetic maps to the reconstructed ones focusing on matching the intensity contrast, the morphology of stellar surface structures, and the photocentre position at two different spectral channels, 1.52 and $1.70 \mu \mathrm{m}$, simultaneously.

Results. We measured the apparent diameter of CL Lac at two wavelengths $(3.299 \pm 0.005$ mas and $3.053 \pm 0.006$ mas at 1.52 and $1.70 \mu \mathrm{m}$, respectively) and recovered the radius $\left(R=307 \pm 41\right.$ and $\left.R=284 \pm 38 R_{\odot}\right)$ using a Gaia parallax. In addition to this, the reconstructed images are characterised by the presence of a brighter area that largely affects the position of the photocentre. The comparison with 3D simulation shows good agreement with the observations both in terms of contrast and surface structure morphology, meaning that our model is adequate for explaining the observed inhomogenities.

Conclusions. This work confirms the presence of convection-related surface structures on an AGB star of Gaia DR2. Our result will help us to take a step forward in exploiting Gaia measurement uncertainties to extract the fundamental properties of AGB stars using appropriate RHD simulations.

Key words. stars: atmospheres - stars: AGB and post-AGB - stars: individual: CL Lac - techniques: interferometric

\section{Introduction}

The Gaia mission (Gaia Collaboration 2016) is an astrometric, photometric, and spectroscopic space-borne mission, which in 2018 delivered high-precision astrometric parameters (i.e. positions, parallaxes, and proper motions) for over one billion sources (Gaia Collaboration 2018). A considerable fraction of the intrinsically detected variable stars are long-period variables (LPVs, of which the survey contains more than 150000 , Holl et al. 2018) with large luminosity amplitudes and variability timescales, adequately sampled by Gaia (Gaia Collaboration 2019). Among LPVs, there are cool luminous evolved stars with low to intermediate mass that reached a critical phase of their evolution at the end of the asymptotic giant branch (AGB). They are characterised by (i) large-amplitude variations in radius, brightness, and temperature of the star; (ii) a strong mass-loss rate $\left(\dot{M}=10^{-6}-10^{-4} \mathrm{M}_{\odot} \mathrm{yr}^{-1}\right.$, De Beck et al. 2010) driven by an interplay between pulsation, dust formation in the extended atmosphere, and radiation pressure on the dust (Höfner \& Olofsson 2018); (iii) an inhomogenous visible surface (produced in the interior and shaped by the top of the convection zone: they travel outwards on timescales ranging from weeks to years (Freytag et al. 2017); and (iv) strong molecular absorption bands in the optically thin region (e.g. Lançon \& Wood 2000) and on the top of the convection-related surface structures.

The concordance of all these properties makes the measurement of accurate stellar parameters and mass-loss rate very complex. In this context, a special emphasis has to be put on the problems with establishing their inter-dependence: a quantitative description of how the mass-loss rate depends on individual 
Table 1. Observational stellar properties and parameters of CL Lac.

\begin{tabular}{cccccccccc}
\hline \hline$G^{(a)}$ & $\mathrm{Bp}^{(a)}$ & $\mathrm{Rp}^{(a)}$ & $\mathrm{K} 2 \mathrm{MASS}^{(b)}$ & $\begin{array}{c}L_{\star}{ }^{(c)} \\
\left(L_{\odot}\right)\end{array}$ & $\begin{array}{c}T_{\mathrm{eff}}{ }^{(d)} \\
(\mathrm{K})\end{array}$ & $\begin{array}{c}p \\
(\mathrm{mas})\end{array}$ & $\begin{array}{c}\sigma_{\varpi}{ }^{(a)} \\
(\mathrm{mas})\end{array}$ & $\begin{array}{c}\sigma_{\varpi} \\
(\mathrm{AU})\end{array}$ & $\begin{array}{c}P_{\mathrm{puls}}{ }^{(e)} \\
(\mathrm{yr})\end{array}$ \\
\hline 8.73 & 12.59 & 7.26 & 2.15 & $4888.0 \pm 8.7$ & 3293 & 1.155 & 0.150 & 0.130 & 1.134 \\
\hline
\end{tabular}

Notes. The first four columns display the G, Bp, Rp, and K photometric colours from Gaia and 2MASS; the two following columns indicate the luminosity $\left(L_{\star}\right)$ and effective temperature $\left(T_{\text {eff }}\right)$; the next three show the parallax $(p)$ with its error $\left(\sigma_{\varpi}\right)$ in mas and in AU; the last column reports the pulsation period $\left(P_{\text {puls }}\right) .{ }^{(a)}$ Gaia DR2 (Gaia Collaboration 2018) ${ }^{(b)}$ 2MASS (Skrutskie et al. 2006) ${ }^{(c)}$ Computed from K2MASS with a bolometric correction of 3.0 ${ }^{(d)}$ The temperature is based on Gaia Bp/Rp photometric colours and was estimated for sources brighter than $G=17$ mag for $3000 \mathrm{~K}<T_{\text {eff }}<10000 \mathrm{~K}$. Due to the degeneracy of the photometric temperature with interstellar extinction, an empirically trained machine learning algorithm was used for external spectroscopic datasets in low-extinction regions in order to derive temperatures (Andrae et al. 2018). However, due to the limited training set for cool stars, photometric temperatures have to be taken with extreme caution for these stars. ${ }^{(e)}$ Jura et al. (1993).

Table 2. Calibrators for CL Lac.

\begin{tabular}{ccccc}
\hline \hline Name & Spectral type & Diameter (mas) & Observing night & Reference \\
\hline HD 210855 & F8V & $\Theta_{\text {UD }}=0.594 \pm 0.060$ & UT 2019Jul28 & JMMC, Bourges et al. (2017) \\
HD 219080 & F1V & $\Theta_{\text {LD }}=0.648 \pm 0.008$ & UT 2019Jul27 and UT 2019Jul28 & Maestro et al. (2013) \\
HD 571 & F5II & $\Theta_{\text {UD }}=0.609 \pm 0.059$ & UT 2019Jul27 & JMMC, Bourges et al. (2017) \\
\hline
\end{tabular}

stellar parameters (and, consequently, how it changes as stars evolve) is essential for models of stellar and galactic chemical evolution (Höfner \& Olofsson 2018).

A noteworthy step forward in trying to find a way to quantitatively recover stellar parameters of AGB stars was proposed by Chiavassa et al. (2018) using Gaia DR2 data and stellar convection simulations (Freytag et al. 2017). The authors showed that the convection-related variability causes photocentre ${ }^{1}$ displacements up to $\approx 11 \%$ of the stellar radius and accounts for a substantial part of the Gaia DR2 parallax error. They suggested that the fundamental properties of AGB stars could be measured directly from Gaia parallax errors. However, a final piece of information was missing, because the convection-related structures were quantified only indirectly (i.e. using photocentre displacement). Only with high angular resolution is it possible to detect the presence of stellar surface inhomogenities. This can be achieved using optical interferometry, which has already proven to be a powerful tool providing amazingly resolved aperture synthesis images of evolved stars (e.g. Chiavassa et al. 2010a, 2017; Montargès et al. 2014, 2016, 2017, 2018; Ohnaka et al. 2016, 2017; Wittkowski et al. 2017; Paladini et al. 2018).

In this work, we show the presence of inhomogeneities on the stellar surface of the AGB star CL Lac, which is part of the sample of Gaia DR2 objects for which Chiavassa et al. (2018) showed that photocentre displacement explains the parallax error bars. The article is structured as follows: Sect. 2 introduces the interferometric observations and data reduction, while Sect. 3 evidences the interpretation of the data with 3D simulation of stellar convection. Section 4 summarises the conclusions and the impact on the determination of AGBs' stellar properties using Gaia parallax errors.

\section{Interferometric observations with MIRC-X at CHARA}

The main objective of this work is to detect the presence of stellar surface inhomogeneities for an object from Gaia's sample used in Chiavassa et al. (2018), and for which the convection-related

1 The intensity-weighted mean of all emitting points tiling the visible stellar surface. variability accounts for a substantial part of the Gaia DR2 parallax error. We observed the LPV AGB star CL Lac with the MIRC-X beam combiner (Kraus et al. 2018; Anugu et al. 2018) and with the VEGA instrument (Mourard et al. 2009) installed at Georgia State University Center for High Angular Resolution Astronomy (CHARA). The CHARA array is located on Mount Wilson, CA, and consists of six 1-m telescopes for a total of 15 baselines ranging in length from 34 to $331 \mathrm{~m}$, resulting in an angular resolution of 0.5 mas in the $H$ band (ten Brummelaar et al. 2005). The observations took place on both UT 2019Jul27 and 2019Jul28. Some stellar properties of CL Lac are reported in Table 1.

\subsection{Data reduction}

We combined the light from all six telescopes to record three calibrated sets of data on each night on CL Lac using MIRC-X in PRISM50 mode, which covers the $H$ band wavelength range $[1.52,1.55,1.58,1.61,1.64,1.67,1.70] \mu \mathrm{m}$. On each night, we recorded three sets of MIRC-X data on CL Lac. Each set consisted of 10-15 min to record fringe data and another $10 \mathrm{~min}$ to record backgrounds and shutter sequences. In between these sets, we observed calibrator stars to calibrate the interferometric measurements. The calibrators and adopted angular diameters are listed in Table 2. In order to verify, we calibrated the calibrators against each other on each night. We saw no evidence for binarity (closure phases consistent with $0^{\circ}$ within their uncertainties). A fit to the calibrator visibilities provided angular diameters consistent with the adopted values.

The data were reduced using the MIRC-X data reduction pipeline v1.2.0 $0^{2}$ to produce calibrated visibilities and closure phases. The MIRC-X detector is susceptible to bias in the bispectrum estimation as pointed out by Basden \& Haniff (2004). The data pipeline corrects for this bispectrum bias with a method similar to the one outlined in Appendix $\mathrm{C}$ of that paper. Figure 1 displays the visibility and closure phase data for all the spectral channels as well as the UV-coverage. The data, and in particular the closure phases, denote the presence of large departure points

\footnotetext{
2 https://gitlab.chara.gsu.edu/lebouquj/mircx_ pipeline.git.
} 

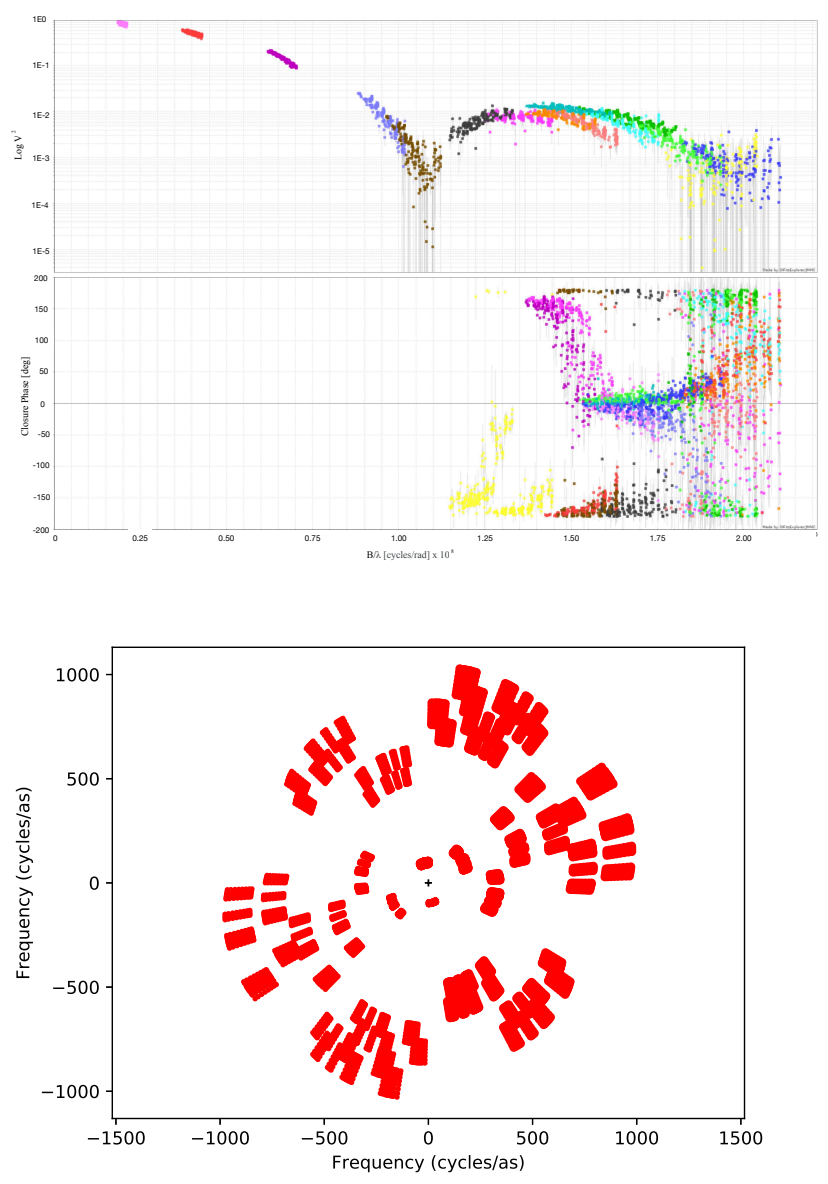

Fig. 1. Top panels: squared visibilities and closure phases for all the data of MIRC-X observations of CL Lac. Visibilities are plotted in a log scale. The different colours indicate different baselines. Bottom panel: UV coverage. The spectral coverage contains wavelengths between 1.52 and $1.70 \mu \mathrm{m}$. The resolution of the interferometer corresponds to a beam of $323.42 \times 323.42 \mathrm{~m}$ telescope, $0.48 \times 0.48 \mathrm{mas}$ and $0.55 \times 0.55$ at 1.52 and $1.70 \mu \mathrm{m}$, respectively.

from the centrosymmetric case (i.e. closure phases not equal to 0 or $\left.\pm 180^{\circ}\right)$. The calibrated OIFITS files are available on the Optical interferometry DataBase ${ }^{3}$.

So as to obtain differential stellar radii in the optical and infrared, in order to retrieve information about the stellar dynamics and opacity run, we performed simultaneous observations with VEGA. We used the MIRC-X beam combiner as a fringe tracker. The longitudinal dispersion correctors were used to compensate for differences in dispersion between the visible and near-infrared. However, no fringes were spotted out in the optical with VEGA. This can be due by the combination of a large object $(>4.5$ mas) and low squared visibilities $(<0.1)$, very close to the limit of the instrumental capabilities. Nevertheless, we cannot definitively conclude on this point because the SNR was significantly lower than the confidence limit of three.

\subsection{Aperture synthesis imaging}

For the image reconstruction, we used the MIRA software package developed by Thiébaut (2008). This package is written in the scientific language yorick ${ }^{4}$. It reads interferometry data in

\footnotetext{
3 http://oidb.jmmc.fr/collection.html?id=

3d64620d-a152-4e75-9bc4-c68735d293b1

4 https://software.1lnl.gov/yorick-doc/
}

the form of oifits files (Pauls et al. 2005), and the reconstructed image is compared to the visibility and closure phase data by means of Fourier transforms and a few additional steps. The pixel values are modulated to minimise a so-called cost function, which is the sum of a regularisation term plus data-related terms $\left(\chi^{2}\right)$. The data terms enforce agreement of the model image with the measured data (visibilities and closure phases). The regularisation term is a distance minimisation between the reconstructed image and an expected image, called the prior.

The data and the regularisation terms must be scaled to maximise the Bayesian evidence (the overall probability of the model integrated over all possible model parameter values). The scaling factor is called the "hyperparameter" $(\mu)$.

We reconstructed images for the spectral channels individually (Fig. A.1) with: 0.2 mas pixel $^{-1}$, a $64 \times 64$-pixel field of view (FOV), a total variation regularisation (Renard et al. 2011), a circular Gaussian prior image with a FWHM equal to half the field of view, and a hyperparameter value of $\mu=5 \times 10^{4}$. This value of $\mu$ is confirmed by the L-curve plot shown in Fig. B.4, which shows the data term $\left(\chi^{2}\right)$ as a function of the hyperparameter $(\mu$, all other image reconstruction parameters are random: pixel size, number of pixels, prior size, number of iterations): one can see that the $\chi^{2}$ has a value close to 2 with $\mu \leq 50000$, and that the value raises by a large amount above. We show here the images convolved with a beam of FWHM $\lambda / 2 B$, meaning two times smaller as the theoretical angular resolution of the interferometer. This is to take advantage of the "super-resolution" effect of image reconstruction with a sparse aperture reported in many works (e.g. Thiébaut 2008). For convenience, the image convolved with a beam of FWHM $\lambda / B$ is also shown in Appendix B (Fig. B.5).

We also reconstructed images using $32 \times 32$ and $128 \times 128$ pixels with the same pixel size (see Fig. B.3), $64 \times 64$ images with different pixel sizes (from 0.1 to 0.4 mas, Fig. B.1), and hyperparameter values (from 5 to $5 \times 10^{6}$, see Fig. B.2), to ensure that the images are not affected by reconstruction artifacts. We also checked that the global appearance of the image is similar when reconstructing a "grey" image (i.e. computing an average across the spectral channels) and a chromatic image (Fig. B.3).

Figure 2 displays two examples of aperture synthesis images at 1.52 and $1.70 \mu \mathrm{m}$. They were convolved with the resolution of the interferometer (Fig. 1, bottom panel). Both images clearly show a brighter area in the south-eastern part of the map that affects the position of the barycentre, which does not correspond to the geometrical one (dashed blue line). The morphology of CL Lac is complex and not centrosymmetric, largely affecting astrometric Gaia measurements (Chiavassa et al. 2018). These stellar surface inhomogenities show a similar behaviour for all the spectral channels but with noticeable changes between 1.52 and $1.70 \mu \mathrm{m}$. Figure 2 (bottom panel) displays large differences (up to $\sim 20 \%$ ), in particular close to the outer stellar disc borders.

\section{Interpreting stellar surface inhomogeneities}

\subsection{Three-dimensional simulations of $A G B$ stars and synthetic images}

We use the radiation-hydrodynamics (RHD) simulation of AGB stars (Freytag et al. 2017) computed with CO $^{5}$ BO1D (Freytag et al. 2012) code. The code solves the coupled non-linear equations of compressible hydrodynamics and non-local radiative energy transfer in the presence of a fixed external spherically symmetric gravitational field on a 3D cartesian grid. The 

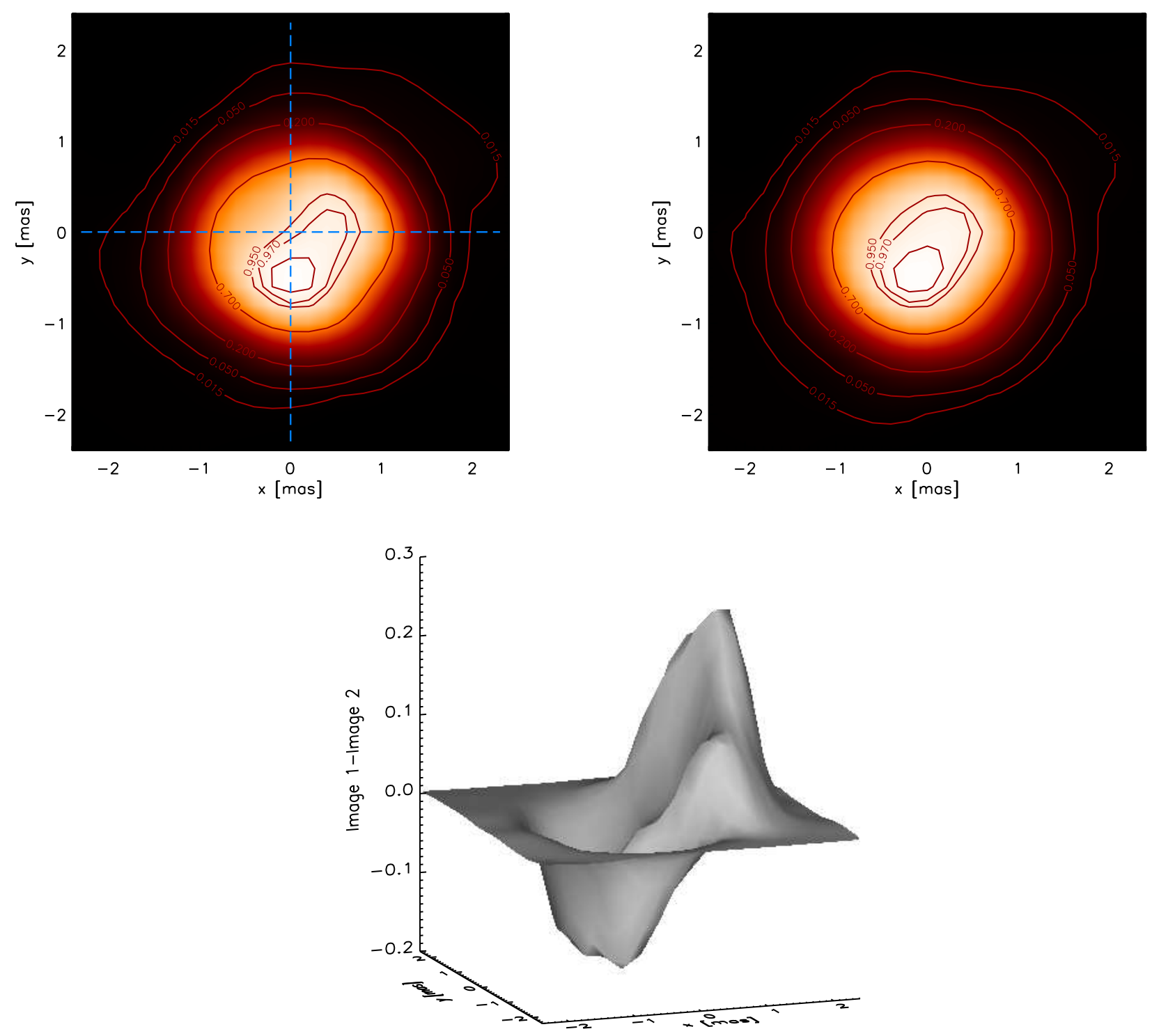

Fig. 2. Top panels: reconstructed images of CL Lac at $1.52 \mu \mathrm{m}$ (left) and $1.70 \mu \mathrm{m}$ (right). They are convolved with the interferometric beam and the intensity is normalised between $[0,1]$. The contour lines correspond to $[0.015,0.05,0.20,0.70,0.95,0.97,0.99]$. East is to the left, north is up. The barycentre of a symmetric star would be expected at $x=0$ and $y=0$, as shown by the blue dashed line. Bottom panel: surface rendering difference between CL Lac images at 1.52 and $1.70 \mu \mathrm{m}$.

averaged stellar parameters of the simulation used in this work are reported in Table 3. The atmosphere in the simulation is characterised by global and small-scale shocks induced by largeamplitude, radial, and fundamental-mode pulsations (Freytag et al. 2017). The pulsation period is extracted with a fit of the Gaussian distribution in the power spectra of the simulations. The shocks contribute to the levitation of material and to the photocentre displacement (Fig. A.5 for the temporal behaviour, Chiavassa et al. 2018). A photocentre displacement of $\sigma_{P}=$ 0.131 AU (Table 3 ) corresponds to $\approx 27 R_{\odot}$ or $\approx 9 \%$ of the stellar radius.

To prepare the comparison with the interferometric data, we computed intensity maps in the same observed spectral channels (Fig. 3) for the 100 snapshots of the RHD simulation. For this purpose, we employed the code OPTIM3D (Chiavassa et al. 2009), which takes into account the Doppler shifts caused by the convective motions. The radiative transfer is computed in detail using pre-tabulated extinction coefficients per unit mass, like for MARCS (Gustafsson et al. 2008) as a function of temperature, density, and wavelength for the solar composition (Asplund et al. 2009). The temperature and density distributions are optimised to cover the values encountered in the outer layers of the RHD simulation.

\subsection{Stellar radius thanks to the Gaia parallax}

The first step of our analysis is the determination of the stellar radius. In order to do this, we used (i) 1D, stationary, hydrostatic models; and (ii) the 3D RHD simulation of Table 3.

For item (i), we selected several models from the MARCSgrid $^{5}$ (Gustafsson et al. 2008) with stellar parameters close to CL Lac (Table 1): effective temperature $T_{\text {eff }}=[2500,2600,2700$, 2800], mass $=1 M_{\odot}, \log g=[-0.5,0.0]$, metallicity $[\mathrm{Fe} / \mathrm{H}]=$ [0.0]. We used the code TurboSpectrum (Plez 2012) to compute intensity profiles from these models in the same observed spectral channels of Fig. 2.

5 http://marcs.astro.uu.se 
Table 3. Parameters of the RHD simulation used in this work.

\begin{tabular}{lccccccccc}
\hline \hline Simulation & $\begin{array}{c}M_{\star}(a) \\
\left(M_{\odot}\right)\end{array}$ & $\begin{array}{c}L_{\star}(a) \\
\left(L_{\odot}\right)\end{array}$ & $\begin{array}{c}R_{\star}(a) \\
\left(R_{\odot}\right)\end{array}$ & $\begin{array}{c}T_{\mathrm{eff}}(a) \\
(\mathrm{K})\end{array}$ & $\begin{array}{c}\log g^{(a)} \\
(\mathrm{cgs})\end{array}$ & $\begin{array}{c}t_{\mathrm{avg}}(a) \\
(\mathrm{yr})\end{array}$ & $\begin{array}{c}P_{\mathrm{puls}}(a) \\
(\mathrm{yr})\end{array}$ & $\begin{array}{c}P^{(b)} \\
(\mathrm{AU})\end{array}$ & $\begin{array}{c}\sigma_{P}{ }^{(b)} \\
(\mathrm{AU})\end{array}$ \\
\hline st28gm05n001 & 1.0 & $5040 \pm 195$ & $306 \pm 18$ & $2790 \pm 59$ & $-0.54 \pm 0.05$ & 25.36 & $1.026 \pm 0.135$ & 0.183 & 0.131 \\
\hline
\end{tabular}

Notes. This simulation is part of the AGB grid of Freytag et al. (2017). The first column shows the simulation name, then the next five columns the stellar parameters such as the mass $\left(M_{\star}\right)$, the average luminosity $\left(L_{\star}\right)$, radius $\left(R_{\star}\right)$, effective temperature $\left(T_{\text {eff }}\right)$, and surface gravity (log $\left.g\right)$. The seventh column displays the stellar simulated time $\left(t_{\text {avg }}\right)$ used to average all the quantities. A solar metallicity is assumed. The eighth and ninth columns report the pulsation period $\left(P_{\text {puls }}\right)$ and the half of the distribution of the pulsation frequencies $\left(\sigma_{\text {puls }}\right)$. Finally, the last two columns are the time-averaged value of the photocentre displacement $P$ and its standard deviation $\left(\sigma_{P}\right)$.

References. ${ }^{(a)}$ Freytag et al. (2017) ${ }^{(b)}$ Chiavassa et al. (2018).

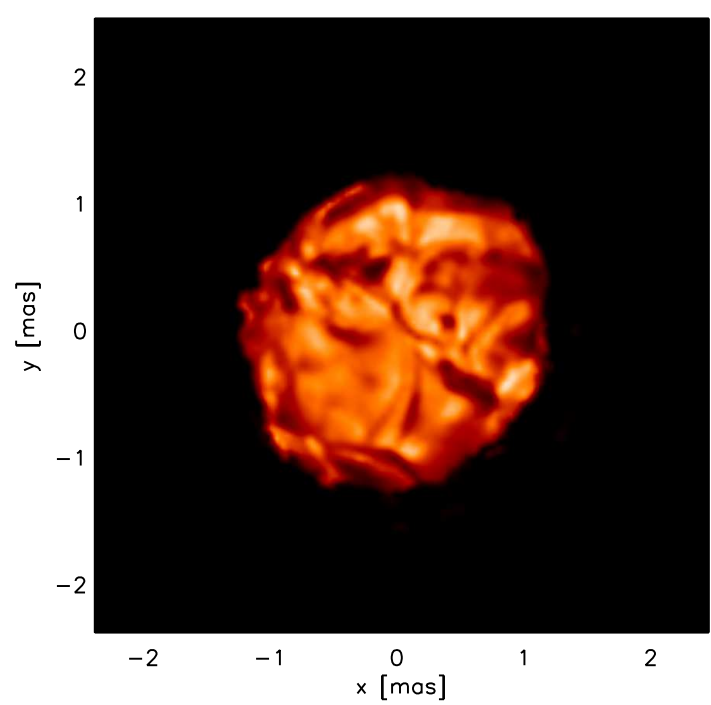

Fig. 3. Synthetic intensity map computed for one snapshot of the RHD simulation of Table 3 at $1.52 \mu \mathrm{m}$. The intensity is normalised between $[0,1]$. East is left, north is up. This image is the non-convolved version of Fig. 7 (top-left panel).

For item (ii), we obtained the 100 azimuthally averaged intensity profiles from the synthetic maps using the method explained by Chiavassa et al. (2009). They were constructed using rings that are regularly spaced in $\mu$ (where $\mu=\cos (\theta)$, with $\theta$ being the angle between the line of sight and the radial direction). Figure 4 shows that the temporal fluctuation from one snapshot to another (green curves) is large, each snapshot being approximatively 23 days apart. Afterwards, we calculated synthetic visibility amplitudes from the different 1D-MARCS models (purple curve) and the 3D temporally averaged (black curve) intensity profiles using the Hankel transform. Finally, we fitted the visibility data from Fig. 1 separately for the two spectral channels at 1.52 and $1.70 \mu \mathrm{m}$ to obtain the apparent diameter of CL Lac. The best-fitting MARCS model has $T_{\text {eff }}=2800 \mathrm{~K}$ and $\log g=-0.5$.

Table 4 reports the $1 \mathrm{D}$ and $3 \mathrm{D}$ stellar diameters as well as the stellar radius at a distance of $d=870 \pm 113$ pc (i.e. inversion of the Gaia parallax, Table 1). The 1D-MARCS model returns apparent radii larger than the $3 \mathrm{D}, \sim 1 \%$ and $5 \%$ at 1.52 and $1.70 \mu \mathrm{m}$, respectively. This is already noticeable from the intensity profiles in Fig. 4. Our result is in qualitative agreement with those obtained by several authors for dwarf and K giant stars (Allende Prieto et al. 2002; Aufdenberg et al. 2005; Bigot et al. 2006; Chiavassa et al. 2010b; Creevey et al. 2012; Kervella et al. 2017). Moreover, wavelength-dependence of the
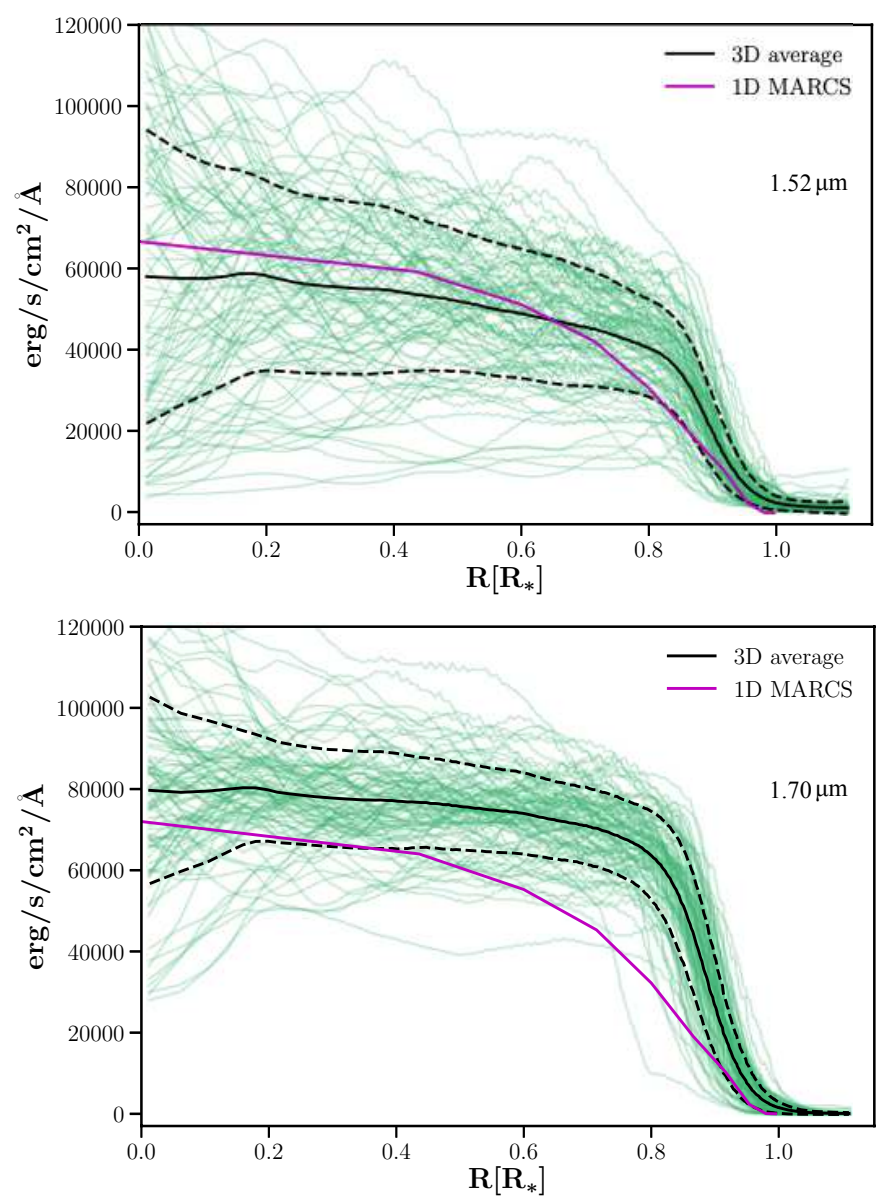

Fig. 4. Azimuthally averaged intensity profiles (green curves) obtained from the intensity map of each simulation snapshot of Table 3. The black curve is the temporally averaged intensity profile, while the dashed black lines denote the $1-\sigma$ temporal fluctuations. The purple line in the $1 \mathrm{D}$ intensity profile from the best-fitting MARCS model (see text).

Table 4. CL Lac apparent diameters obtained with the best-fitting 1D-MARCS model, $\Theta_{1 \mathrm{D}}$, and the one from the $3 \mathrm{D}$ temporally averaged intensity profile (Fig. 4, black curve), $\Theta_{3 \mathrm{D}}$.

\begin{tabular}{ccccc}
\hline $\begin{array}{c}\text { Wavelength } \\
(\mu)\end{array}$ & $\begin{array}{c}\Theta_{1 \mathrm{D}} \\
(\mathrm{mas})\end{array}$ & $\begin{array}{c}R_{\star} \\
\left(R_{\odot}\right)\end{array}$ & $\begin{array}{c}\Theta_{3 \mathrm{D}} \\
(\mathrm{mas})\end{array}$ & $\begin{array}{c}R_{\star} \\
\left(R_{\odot}\right)\end{array}$ \\
\hline 1.52 & $3.332 \pm 0.005$ & $310 \pm 41$ & $3.299 \pm 0.005$ & $307 \pm 41$ \\
1.70 & $3.220 \pm 0.004$ & $300 \pm 40$ & $3.053 \pm 0.006$ & $284 \pm 38$ \\
\hline
\end{tabular}



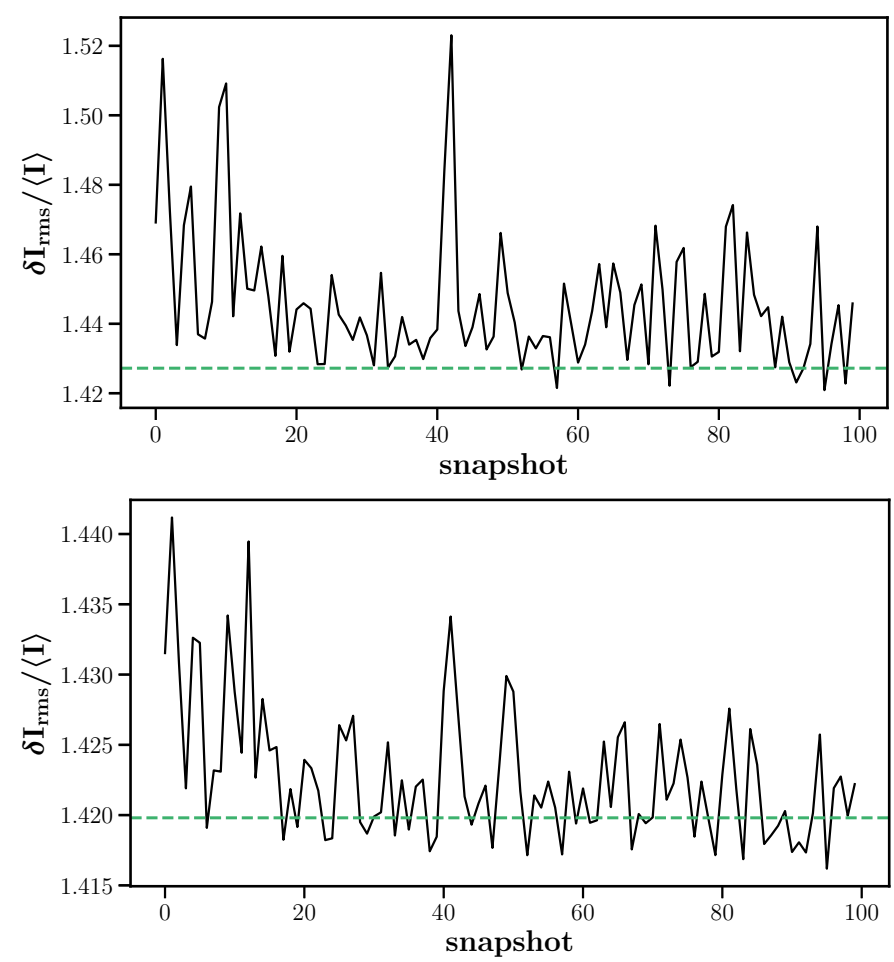

Fig. 5. Fluctuations at $1.52 \mu \mathrm{m}$ (top) and $1.70 \mu \mathrm{m}$ (bottom) for all the snapshots of the RHD simulation of Table 3 after being corrected for limb-darkening and convolved with the interferometric beam. The horizontal dashed line displays the contrasts measured in the reconstructed images of Fig. 2: 1.424 at $1.52 \mu \mathrm{m}$ and 1.423 at $1.70 \mu \mathrm{m}$.

radius is noticeable: this is a signature of different contributions of molecular absorption (Wittkowski et al. 2016), even if the interpretation remains difficult at the actual resolution of our observations.

To sum up, the RHD simulation used here is the closest available to CL Lac in terms of

1. stellar parameters with, in particular, a very similar radius $\left(R_{\star}=306 \pm 18\right.$, Table 3 ) with respect to the value we found (Table 4) and pulsation period;

2. photocentre variability in the Gaia $G$ band $\left(\sigma_{P}=0.131\right.$, Table 3) caused by convection-related structures that accounts, as demonstrated by Chiavassa et al. (2018), for a substantial part of the CL Lac parallax error $\left(\sigma_{\varpi}=0.130\right.$, Table 1).

It has to be noted that the $T_{\text {eff }}$ based on Gaia $\mathrm{Bp} / \mathrm{Rp}$ photometric colours from Table 1 is almost $500 \mathrm{~K}$ larger than the 1D MARCS and 3D RHD simulation, that, in turn, have overlapping values within error bars. However, due to the limited training set for cool stars in Gaia actual DR2, photometric temperatures have to be taken with extreme caution for these stars.

\subsection{Comparison with the $3 D$ RHD simulation}

In this section, we compare the synthetic maps to the aperture synthesis imaging. As a first step, we correct the limbdarkening (LD) effect dividing both the synthetic and reconstructed images. We proceeded as follows: (i) we created an LD model image $\left(64 \times 64\right.$ pixels and 0.2 mas pixel $^{-1}$ resolution) with the same FOV as the reconstructed image using the $3 \mathrm{D}$ temporally averaged profile of Fig. 4; (ii) we rebinned the resolution of 0.2 mas pixel $^{-1}$ the $3 \mathrm{D}$ synthetic maps so that they effectively possess the same FOV and resolution as the reconstructed
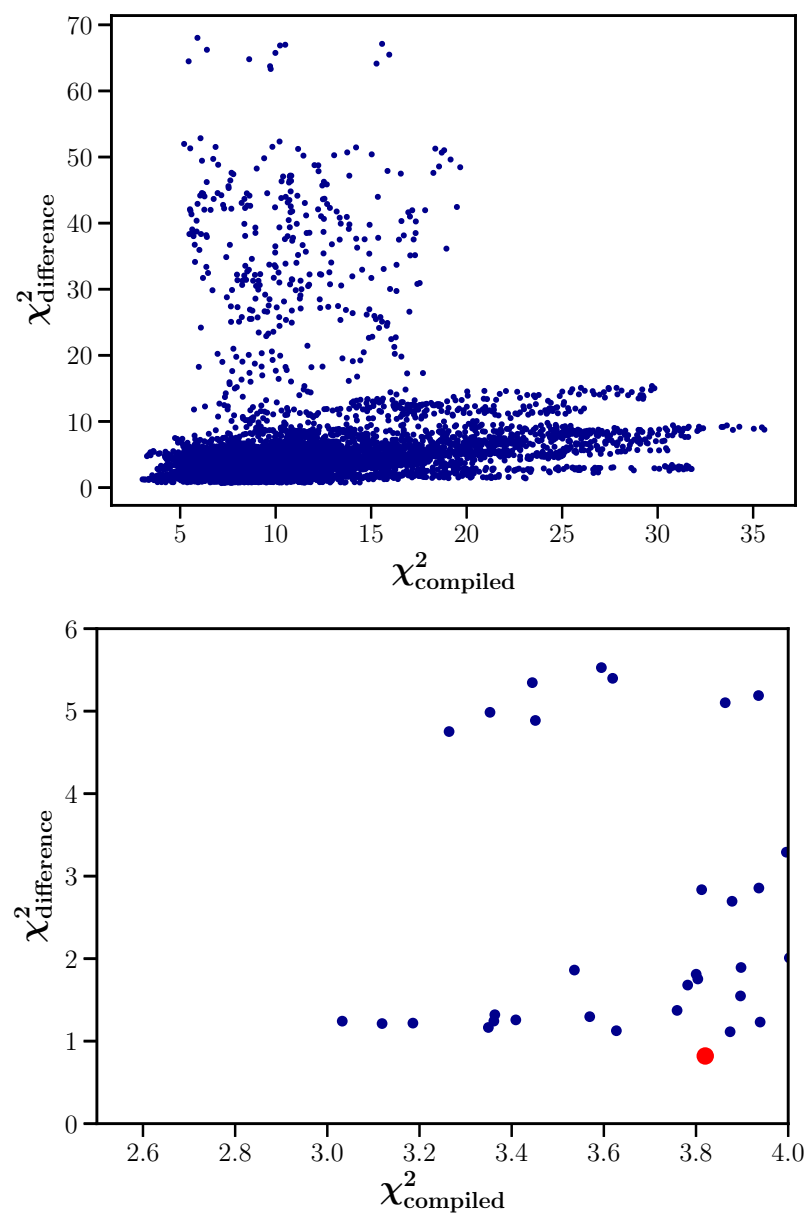

Fig. 6. Top panel: compiled $\chi_{\text {compiled }}^{2}=\chi_{\text {contrast }}^{2}+\chi_{\text {morphology }}^{2}+\chi_{\text {photocentre }}^{2}$ versus $\chi_{\text {difference }}^{2}=\Delta_{\text {reconstructed }}-\Delta_{3 \mathrm{D}}$ (see text for details) for all the snapshots and rotation angles considered (blue points) for the comparison with the reconstructed images. Bottom panel: enlargement within the region where the best matching snapshot and rotation angle is chosen (large red point).

images; and (iii) we convolved them to the resolution of the interferometric observations (Fig. 1, bottom panel) for both spectral channels at 1.52 and $1.70 \mu \mathrm{m}$.

To have strong constraints on the RHD simulation, we simultaneously inspected three different quantities for each map: [1] the intensity contrast in the maps; [2] the morphology of the surface structures; and [3] the position of the photocentre. We performed a minimisation procedure for each item to obtain the normalised $\chi_{\text {compiled }}^{2}=\chi_{\text {contrast }}^{2}+\chi_{\text {morphology }}^{2}+\chi_{\text {photocentre }}^{2}$.

Starting from point [1], we divided pixel by pixel reconstructed images, and we convolved 3D synthetic maps by the LD one and used the definition of Tremblay et al. (2013) to estimate the intensity contrast as $\delta I_{\mathrm{rms}} /\langle I\rangle$. The contrast depends on the radial cut chosen (i.e. the maximum radius adopted from the centre of the star from which outer pixels are not considered, Montargès et al. 2018). For CL Lac, we chose to cut at 0.80 stellar radii to avoid outer areas with rapid and steep increase. Figure 5 shows that several snapshots have very close values of contrast with respect to observations for both wavelength channels. The best matching 3D synthetic snapshot is the one with the closest intensity contrast with respect to the reconstructed images.

Furthermore, we concentrated our analysis on the morphology of the surface structures (point [2]). To account for the 

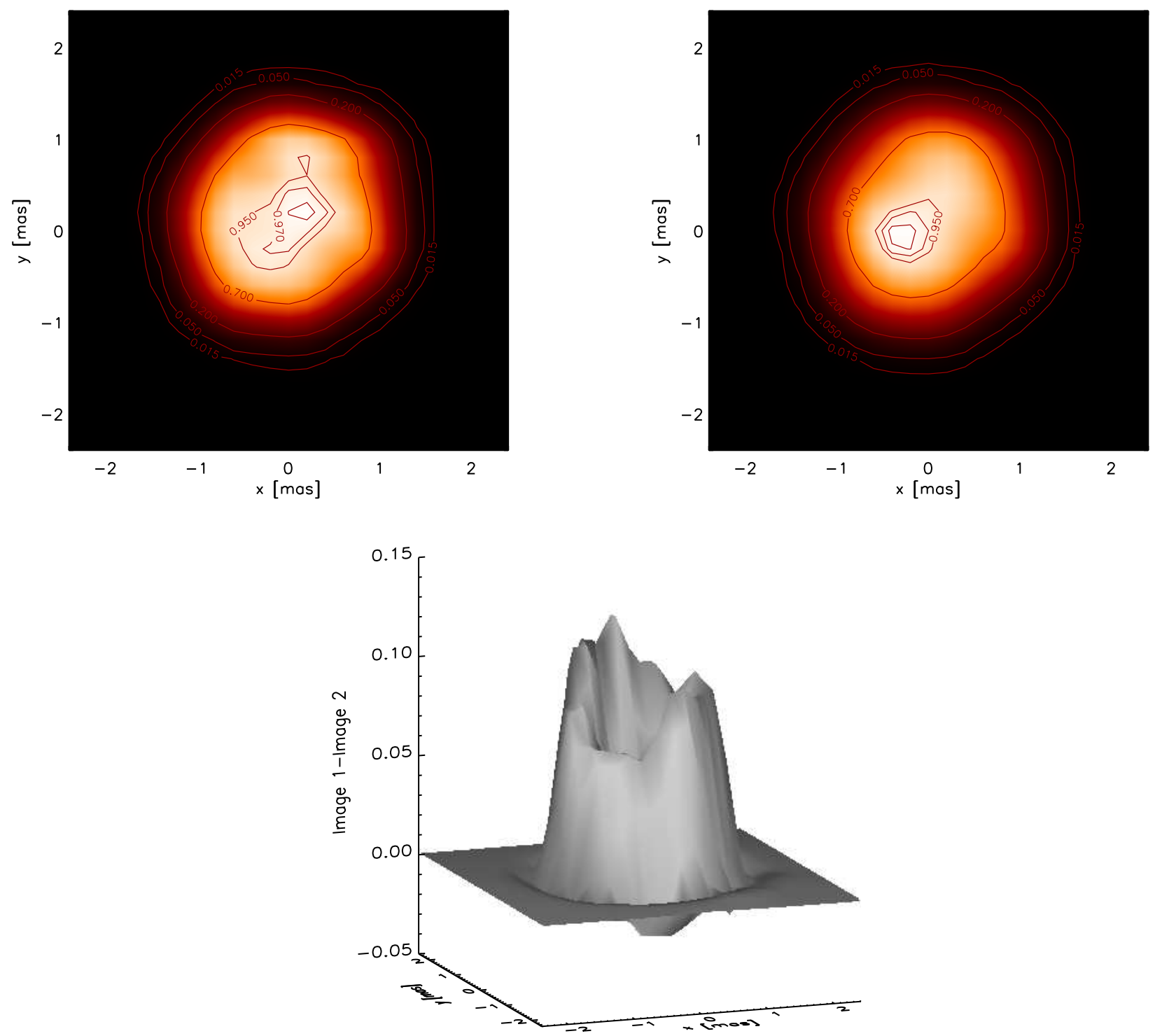

Fig. 7. Top panels: synthetic intensity maps computed for the best matching RHD simulation of Table 3 at 1.52 (left) and 1.70 (rigth) $\mu$ m. The intensity is normalised between [0,1]. The images, $64 \times 64$ pixels with 0.2 mas pixel $^{-1}$, were convolved with the interferometric beam (Fig. 1). The contour lines are the same as in Fig. 2. East is left, while north is up. Bottom panel: surface rendering difference between the synthetic images at 1.52 and $1.70 \mu \mathrm{m}$ (right).

unknown orientation of CL Lac with respect to the synthetic maps in the sky, we rotated the latter every $5^{\circ}$ around its centre, and we calculated the minimum difference with the reconstructed images. Several snapshots were found to be very similar to the observed images, but, as expected, none matched perfectly.

Finally, we computed the position of the photocentre (point [3]) for each map and for the reconstructed images as the intensity-weighted mean of the $x-y$ positions of all emitting points tiling the visible stellar surface (Eqs. (1) and (2) from Chiavassa et al. 2018). Then, we found the smallest difference between the photocentre in the observations and the synthetic maps. Among the different contributors to the $\chi_{\text {compiled }}^{2}$, the morphology of the surface structures $\left(\chi_{\text {morphology }}^{2}\right)$ is the most dominant.

In addition to these three points, we also considered the difference between the reconstructed images at 1.52 and $1.70 \mu \mathrm{m}$ $\left(\Delta_{\text {reconstructed }}=\right.$ image $_{1.52}-$ image $_{1.70}$, bottom panel of Fig. 2$)$ and for the simulation images $\left(\Delta_{3 \mathrm{D}}=\right.$ image $3 \mathrm{D}_{1.52}-$ image $3 \mathrm{D}_{1.70}$, bottom panel of Fig. 7). We aimed to have the closest difference $(\Delta)$ between the two cases, and we defined $\chi_{\text {difference }}^{2}=$ $\Delta_{\text {reconstructed }}-\Delta_{3 \mathrm{D}}$. This approach gives an additional and important wavelength dependence constraint for the simulation. In the end, the best matching snapshot is the one with the combination of the smallest $\chi_{\text {compiled }}^{2}$ versus $\chi_{\text {difference }}^{2}$, with a higher weight for the latter. Figure 6 shows all the snapshots and all the different rotation angles considered, while Fig. 7 displays the best matching snapshot and rotation angle: the best combination returns $\chi_{\text {compiled }}^{2}=3.8$ and $\chi_{\text {difference }}^{2}=0.8$.

Our 3D RHD simulation shows a good agreement with the observations both in terms of contrast a surface structures and wavelength dependence, meaning that it is adequate for explaining the inhomogenities observed in the reconstructed maps. This definitively confirms the presence of convection-related surface structures on the same Gaia DR2 object for which Chiavassa et al. (2018) proved that photocentre displacement explains the parallax error bars. 


\section{Discussion and conclusions}

Stellar dynamics induce an intrinsic noise that increases the measurement uncertainty of the parallax of AGB stars in Gaia DR2 (Chiavassa et al. 2018), among which there is the star CL Lac. However, the effect of convection-related surface structures was estimated only indirectly using the displacement of the photocentre $\left(\sigma_{P}\right)$. In addition to this, the authors showed also a clear correlation between stellar parameters (surface gravity and pulsation period) and the standard deviation of the photocentre movements: 3D simulations with a lower surface gravity (i.e. more extended atmospheres) return larger excursions of the photocentre.

Thanks to optical interferometry, we managed, in this work, to discover the presence of stellar inhomogenities on the AGB CL Lac. Its morphology is complex, not centrosymmetric, and likely affects astrometric Gaia measurements. Using stateof-the-art 3D global simulations of stellar convection with $\mathrm{CO}^{5} \mathrm{BOLD}$, we were able to interpret them as a consequence of small- and global-scale shocks that contribute to the levitation of material. This definitively confirms the presence of convectionrelated surface structures on the same Gaia DR2 object for which Chiavassa et al. (2018) discussed the photocentre displacement.

Our result will help us to make a step forward in exploiting Gaia measurement uncertainties using appropriate RHD simulations. The long-term aim is to uniquely extract the fundamental properties of AGB stars directly from Gaia errors and make it possible to systematically study the properties of convection in stars other than the Sun. These properties are paramount to understanding the physics of these stars: for example, the surface gravity controls the size of granules that, in turn, contribute to the stellar photometric variations. Similarly, the pulsation period provides important information about stellar (mean) interior as well as global shocks induced by large-amplitude, radial, and fundamental-mode pulsations.

To achieve our goal, a series of steps are required. We list them here:

1. An additional study of the photometric colours from $\mathrm{Bp}$ and Rp photometric systems; Chiavassa et al. (2011) showed that red supergiant stars ${ }^{6}$ have magnitude excursions up to 0.28 and 0.13 magnitudes in $\mathrm{Bp}$ and $\mathrm{Rp}$, respectively. This impacts the determination of their parameters. We expected similar (or higher) values for AGBs.

2. One limitation of the existing model grid (Freytag et al. $2017)$ is the restriction to $1 M_{\odot}$. A significantly larger RHD simulation grid with a larger range of stellar masses is necessary to explore the large number of AGBs in Gaia (Holl et al. 2018).

3. With Gaia DR2, the mean number of measurements for each source amounts to $\approx 26$ (Mowlavi et al. 2018). Finally, when Gaia DR4 is available in 2022, the number of measurements will increase to 70-80, possibly changing the parallax error. Additionally, DR4 will provide time-dependant measurements allowing a direct and more precise comparison with the time-dependent RHD simulations.
Acknowledgements. This work is based upon observations obtained with the Georgia State University Center for High Angular Resolution Astronomy Array at Mount Wilson Observatory. The CHARA Array is supported by the National Science Foundation under Grant No. AST-1636624 and AST-1715788. Institutional support has been provided from the GSU College of Arts and Sciences and the GSU Office of the Vice President for Research and Economic Development. MIRC-X received funding from the European Research Council (ERC) under the European Union's Horizon 2020 research and innovation programme (Grant No. 639889). We also thank the grants NSF 1506540 and NASANNX16AD43G. This work has made use of the MIRA software, kindly developed by E. Thiébaut who we thank for his great contribution to the community.

\section{References}

Allende Prieto, C., Asplund, M., García López, R. J., \& Lambert, D. L. 2002, ApJ, 567, 544

Andrae, R., Fouesneau, M., Creevey, O., et al. 2018, A\&A, 616, A8

Anugu, N., Le Bouquin, J.-B., Monnier, J. D., et al. 2018, SPIE Conf. Ser., 10701, 1070124

Asplund, M., Grevesse, N., Sauval, A. J., \& Scott, P. 2009, ARA\&A, 47, 481

Aufdenberg, J. P., Ludwig, H.-G., \& Kervella, P. 2005, ApJ, 633, 424

Basden, A. G., \& Haniff, C. A. 2004, MNRAS, 347, 1187

Bigot, L., Kervella, P., Thévenin, F., \& Ségransan, D. 2006, A\&A, 446, 635

Bourges, L., Mella, G., Lafrasse, S., et al. 2017, VizieR Online Data Catalog: II/346

Chiavassa, A., Plez, B., Josselin, E., \& Freytag, B. 2009, A\&A, 506, 1351

Chiavassa, A., Lacour, S., Millour, F., et al. 2010a, A\&A, 511, A51

Chiavassa, A., Collet, R., Casagrande, L., \& Asplund, M. 2010b, A\&A, 524, A93

Chiavassa, A., Pasquato, E., Jorissen, A., et al. 2011, A\&A, 528, A120

Chiavassa, A., Norris, R., Montargès, M., et al. 2017, A\&A, 600, L2

Chiavassa, A., Freytag, B., \& Schultheis, M. 2018, A\&A, 617, L1

Creevey, O. L., Thévenin, F., Boyajian, T. S., et al. 2012, A\&A, 545, A17

De Beck, E., Decin, L., de Koter, A., et al. 2010, A\&A, 523, A18

Freytag, B., Steffen, M., Ludwig, H.-G., et al. 2012, J. Comput. Phys., 231, 919

Freytag, B., Liljegren, S., \& Höfner, S. 2017, A\&A, 600, A137

Gaia Collaboration (Prusti, T., et al.) 2016, A\&A, 595, A1

Gaia Collaboration (Brown A. G. A., et al.) 2018, A\&A, 616, A1

Gaia Collaboration (Eyer, L., et al.) 2019, A\&A, 623, A110

Gustafsson, B., Edvardsson, B., Eriksson, K., et al. 2008, A\&A, 486, 951

Höfner, S., \& Olofsson, H. 2018, A\&ARv, 26, 1

Holl, B., Audard, M., Nienartowicz, K., et al. 2018, A\&A, 618, A30

Jura, M., Yamamoto, A., \& Kleinmann, S. G. 1993, ApJ, 413, 298

Kervella, P., Bigot, L., Gallenne, A., \& Thévenin, F. 2017, A\&A, 597, A137

Kraus, S., Monnier, J. D., Anugu, N., et al. 2018, SPIE Conf. Ser., 10701, 1070123

Lançon, A., \& Wood, P. R. 2000, A\&AS, 146, 217

Maestro, V., Che, X., Huber, D., et al. 2013, MNRAS, 434, 1321

Montargès, M., Kervella, P., Perrin, G., et al. 2014, A\&A, 572, A17

Montargès, M., Kervella, P., Perrin, G., et al. 2016, A\&A, 588, A130

Montargès, M., Chiavassa, A., Kervella, P., et al. 2017, A\&A, 605, A108

Montargès, M., Norris, R., Chiavassa, A., et al. 2018, A\&A, 614, A12

Mourard, D., Clausse, J. M., Marcotto, A., et al. 2009, A\&A, 508, 1073

Mowlavi, N., Lecoeur-Taïbi, I., Lebzelter, T., et al. 2018, A\&A, 618, A58

Ohnaka, K., Weigelt, G., \& Hofmann, K. H. 2016, A\&A, 589, A91

Ohnaka, K., Weigelt, G., \& Hofmann, K. H. 2017, Nature, 548, 310

Paladini, C., Baron, F., Jorissen, A., et al. 2018, Nature, 553, 310

Pauls, T. A., Young, J. S., Cotton, W. D., \& Monnier, J. D. 2005, PASP, 117, 1255

Plez, B. 2012, Astrophysics Source Code Library [record ascl: 1205. 004]

Renard, S., Thiébaut, E., \& Malbet, F. 2011, A\&A, 533, A64

Skrutskie, M. F., Cutri, R. M., Stiening, R., et al. 2006, AJ, 131, 1163

ten Brummelaar, T. A., McAlister, H. A., Ridgway, S. T., et al. 2005, ApJ, 628, 453

Thiébaut, E. 2008, SPIE Conf, Ser., 7013, 70131I

Tremblay, P. E., Ludwig, H. G., Freytag, B., Steffen, M., \& Caffau, E. 2013, A\&A, 557, A7

Wittkowski, M., Chiavassa, A., Freytag, B., et al. 2016, A\&A, 587, A12

Wittkowski, M., Abellán, F. J., Arroyo-Torres, B., et al. 2017, A\&A, 606, L1

6 Massive $\left(M>8 M_{\odot}\right.$, Höfner \& Olofsson 2018), cool evolved stars with stellar parameters close to AGBs. 


\section{Appendix A: Reconstructed images for individual spectral channels}

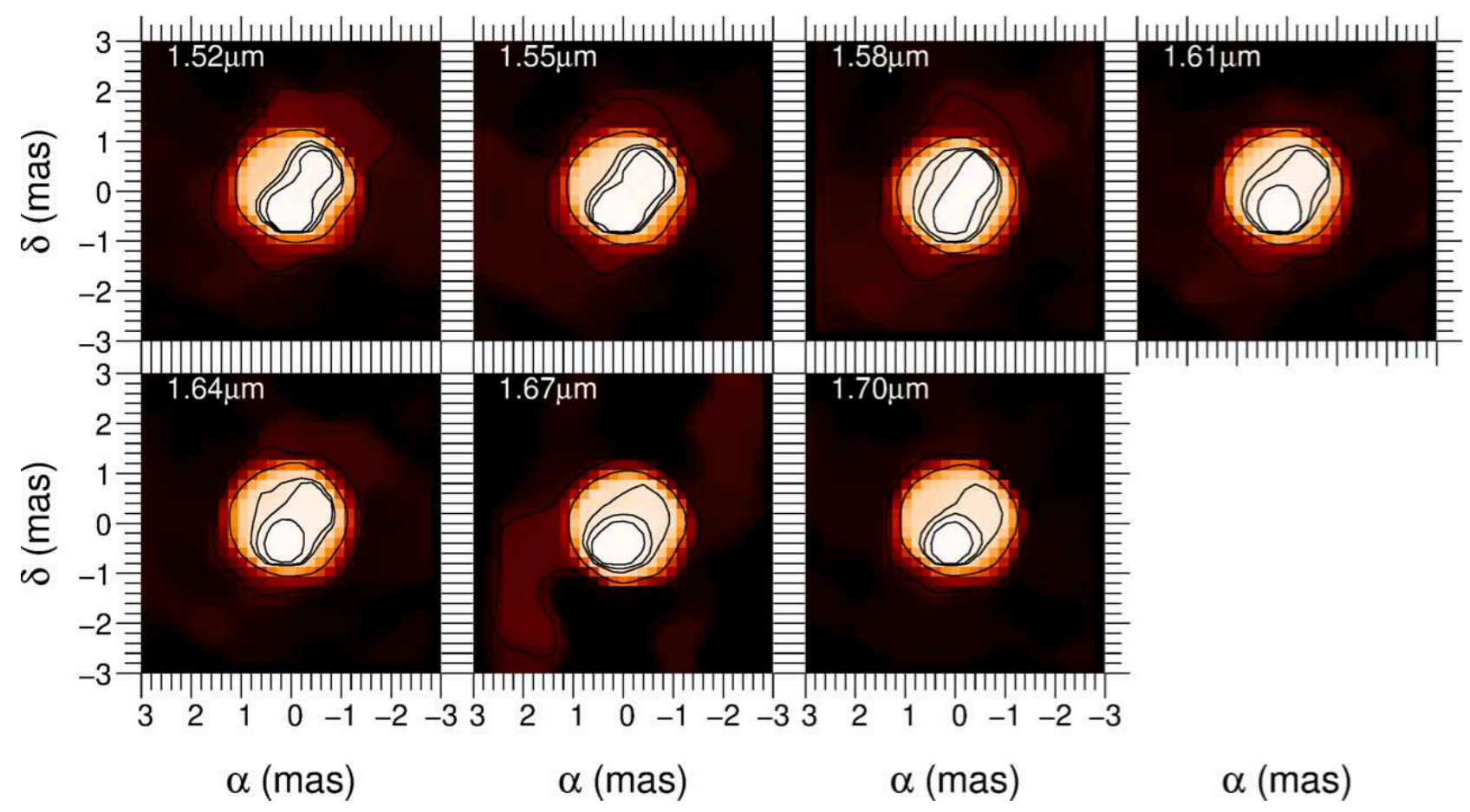

Fig. A.1. Wavelength dependence of the reconstructed images. The intensity is normalised between $[0,1]$, and the contour lines are the same as in Fig. 2. These images have not been convolved with the interferometric beam.

\section{Appendix B: How robust are our images?}

We present here a subset of all the images that were reconstructed on CL Lac with the MIRA software to illustrate the robustness of the features discussed in this work. Image reconstruction with optical interferometry is known to have flaws due to the sparse sampling of the measurements, and also due to the intrinsically non-linear properties of the available interferometric observables (i.e. squared visibilities and closure phases). Therefore, we tested several of the input parameters of MIRA code, namely the pixel size (Fig. B.1), the value of the hyperparameter $\mu$ (Fig. B.2), and the FOV (i.e. changing the number of pixels, Fig. B.3).

Figure B.1 shows that the image behaviour does not change drastically as a function of the pixel size: the disc of the star is the same size, and the bright area in the south-east is always located in the same place. We chose a pixel size of 0.2 mas. Then, Fig. B. 2 displays that too low $\mu$ values $(\leq 10000)$ return inhomogenous images, while too large values $(\geq 100000)$ tend to flatten the surface structures. We selected a median value of $\mu=50000$. Finally, Fig. B.2 indicates that changing the image FOV does not affect its appearance.

Moreover, it has to be noted that the resolution of images reconstructed with MiRA is not necessarily $\lambda / B$, where $B$ is the largest baseline of the interferometric beam. Depending on the signal-to-noise and UV-plane coverage of the data, a degree of super-resolution (up to a factor of 2-3) is possible. In Fig. B.5, we show two examples at $1.52 \mu \mathrm{m}$ with other convolutions, namely an interferometric beam of FWHM $\lambda /(B)$ and $\lambda /(4 B)$. These images have to be compared with those in Fig. 2.

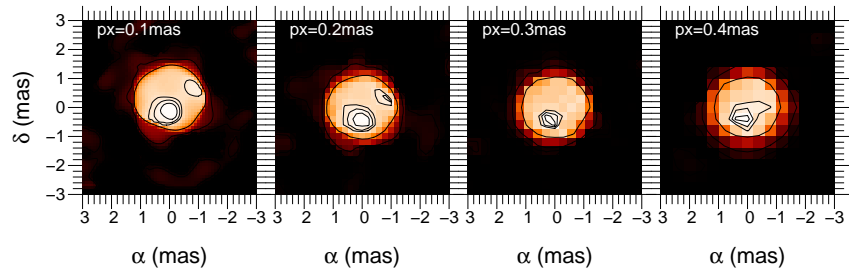

Fig. B.1. Dependence of image appearance on the value of pixel size. The intensity is normalised between $[0,1]$ and the contour lines are the same as in Fig. 2. These images were not convolved with the interferometric beam.

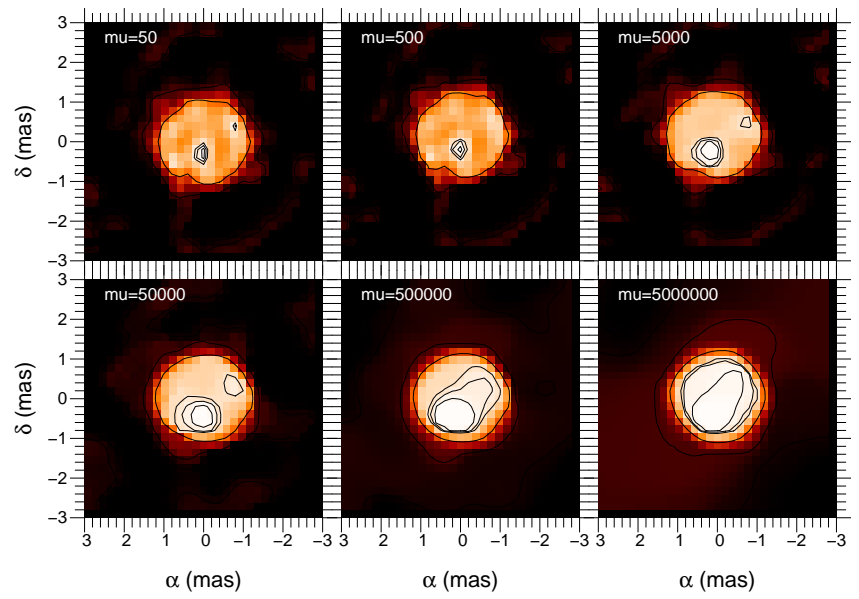

Fig. B.2. Dependence of image appearance on the value of the hyperparameter $\mu$. The intensity is normalised between $[0,1]$ and the contour lines are the same as in Fig. 2. These images were not convolved with the interferometric beam. 


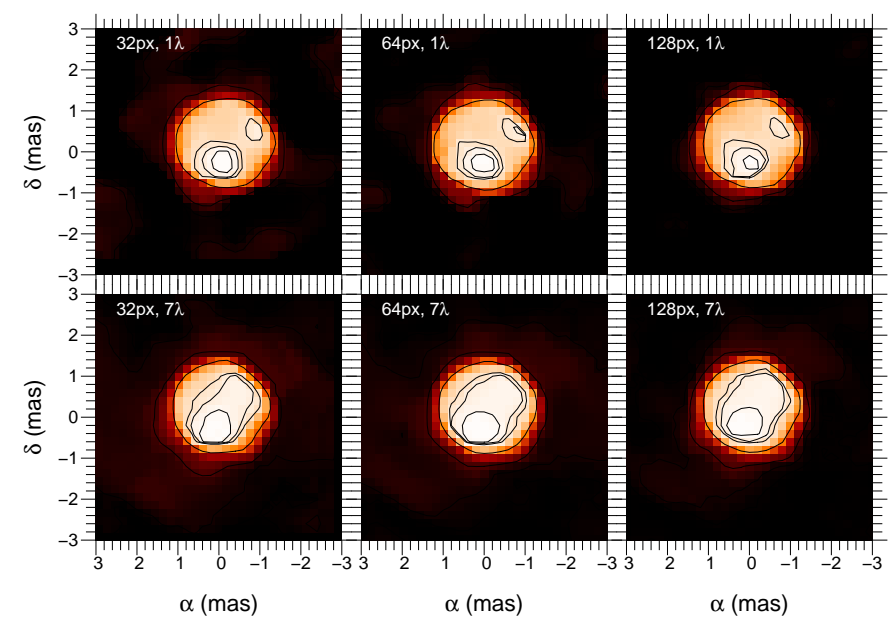

Fig. B.3. Dependence of image appearance on the FOV. Top: grey images (i.e. reconstructed with all $\lambda$ together). Bottom: chromatic image computed with the median of all $\lambda$-reconstructed images. The intensity is normalised between $[0,1]$ and the contour lines are the same as in Fig. 2. These images were not convolved with the interferometric beam.

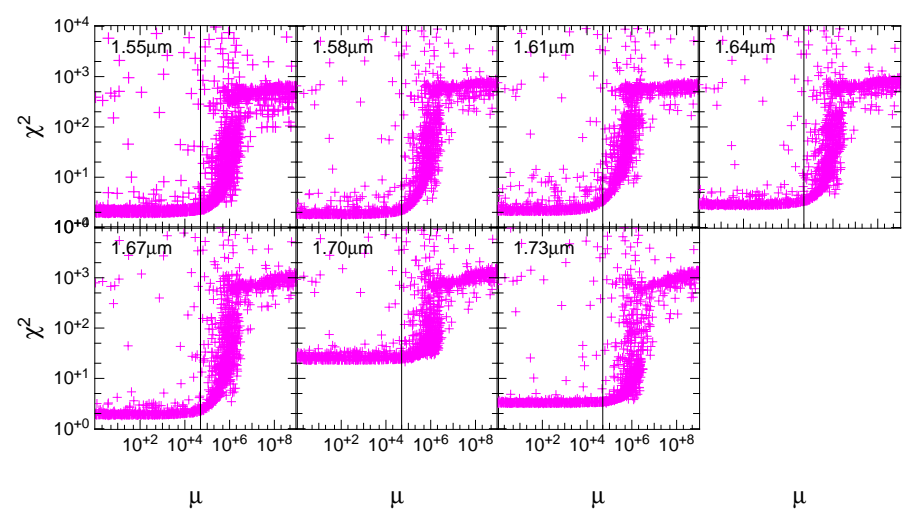

Fig. B.4. L-curve plot: data term $\left(\chi^{2}\right)$ as a function of the hyperparameter $(\mu$, all other image reconstruction parameters are random: pixel size, number of pixels, prior size, number of iterations).
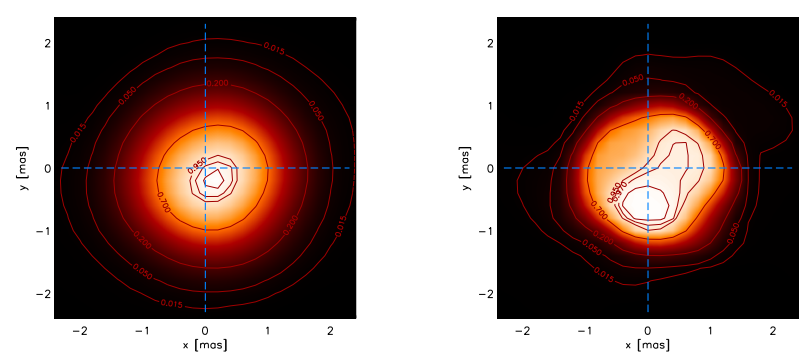

Fig. B.5. CL Lac reconstructed images convolved with an interferometric beam of FWHM $\lambda / B$ (left panel) and FWHM $\lambda / 4 B$ (right panel). The intensity is normalised between $[0,1]$ and the contour lines are the same as in Fig. 2. 